\title{
Periodontal defects after third molar surgery
}

\author{
What is the risk of having periodontal defects on the distal of the mandibular \\ second molar after third molar removal?
}

\begin{abstract}
Richardson DT, Dodson TB. Risk of periodontal defects after third molar surgery: an exercise in evidence-based clinical decisionmaking. Oral Surg Oral Med Oral Pathol Oral Radiol Endod 2005; 100:133-137
\end{abstract}

Data sources Medline was used to source studies for the review. Study selection Prospective studies were selected that had been published in English and had at least 6 months' follow-up data, which facilitated comparison of pre- and postoperative clinical periodontal measures of attachment levels (AL) or probing depths $(\mathrm{PD})$ at the distal of the second molar.

Data extraction and synthesis Perioperative changes in $\mathrm{AL}$ or PD were recorded. For studies reporting mean data, pre-operative and long-term follow-up measures were compared: changes in PD or AL $>2 \mathrm{~mm}$ were considered clinically significant. For subject- or tooth-specific data, periodontal status was recorded as disease present (AL $>2 \mathrm{~mm}$; $\mathrm{PD}>4 \mathrm{~mm}$ ) or disease absent. Pre- and postoperative conditions were compared using $2 \times 2$ tables to estimate number-needed-to-treat (NNT) or number needed to harm (NNH).

Results Eight studies (two prospective cohort studies and six randomised controlled trials) met the inclusion criteria. Mean data were recorded in five studies which, overall, showed clinically insignificant changes in AL/PD at the end of follow-up. Four studies, with sample sizes ranging from 10-24, allowed calculation of NNT and NNH. Where periodontal disease was present preoperatively, NNT ranged from 3-10. Periodontal health status worsened after third molar removal in $48 \%$ of cases, giving a NNH of 2 .

Conclusions No clinically significant changes in AL or PD were identified after third molar removal. In subjects who pre-operatively had a healthy second molar periodontium, there is an increased risk of worsening AL or PD after third molar surgery.

\section{Commentary}

Given the frequency with which third molars are removed and the observation of periodontal defects on distal surfaces of second molar teeth, it makes a great deal of sense to see whether the two are related. Implicit in this statement are a number of questions. The removal of the lower third molar in people who have existing periodontal disease could make matters worse; it could be associated with an improvement; or it could simply be irrelevant. The possible outcomes in a previously periodontally healthy patient are limited to either deterioration or no change.

Without doubt, the modern gold standard of evidence is the systematic review, referred to here as a "critically appraised topic" (CAT). The authors, however, have chosen to formulate just one question and as a result it has been difficult to formulate an overall answer. Systematic reviews should be both comprehensive and critical but here the authors restricted their search to Medline using Ovid. As a result, the review is unlikely to be truly comprehensive: this restriction may have excluded much relevant literature, particularly as there was no strategy for searching by hand the extensive oral surgery and periodontal literature available.

Of real concern is the lack of any critical review. Publications were considered if they met inclusion criteria, ie, prospective design; 6 months' follow-up; and inclusion of measurements of PD or AL. There does not appear to have been any evaluation of the quality of each paper cited. Issues such as baseline sample sizes, randomisation procedures, examiner calibration, examiner bias and completeness of follow-up was not discussed, even though the effects of poor-quality trials are well-documented in the literature. ${ }^{1,2}$

The authors attempted to combine the data from the selected studies in summary tables. The studies are, however, remarkably variable in design and this heterogeneity calls into question the conclusions reached. For example, the varied experimental therapies used meant that only the small control groups could be included in this review.

So, in spite of some interesting calculations of NNT or NNH, is it possible to glean any useful information from this CAT? It is increasingly thought that a deficient host response to the plaque biofilm is more important for the development of periodontal disease than are local factors. The statement that, "one should be circumspect in removing third molars in healthy periodontal patients" is probably sound and is in line with guidelines from the UK National Institute for Clinical Excellence. ${ }^{3}$ Nevertheless, however sensible they seem, the statements provided in this CAT should be interpreted with some caution.

\section{Graham J Smart}

Specialist in Periodontics, London and Oxford, and Postgraduate Dental Tutor for Buckinghamshire, UK

1. Moher D, Jones A, Cook DJ, et al. Does quality of reports of randomised trials affect estimates of intervention efficacy reported in meta-analyses? Lancet 1998, 352:609-613

2. Schulz KF, Chalmers I, Hayes RJ, Altman DG. Empirical evidence of bias: dimensions of methodological quality associated with estimates of treatment effects in controlled trials. J Am Med Assoc 1995; 273:408-412.

3. National Institute for Clinical Excellence. Guidance on the Extraction of Wisdom Teeth. Technology Appraisals Guid ance No. 1. London: National Institute for Clinical Excellence; 2000.

Evidence-Based Dentistry (2006) 7, 46.

doi:10.1038/sj.ebd.6400406
Address for correspondence: Daniel T Richardson, Department of Oral and Maxillofacial Surgery, Massachusetts General Hospital, 55 Fruit Street, Warren 1201, Boston MA 02114, USA. E-mail: dtrichardson@partners.org. 